\title{
Error analysis of regularized least-square regression with Fredholm kernel
}

\author{
Yanfang Tao ${ }^{\mathrm{a}}$, Peipei Yuan ${ }^{\mathrm{b}}$, Biqin Song ${ }^{\mathrm{c}, *}$ \\ ${ }^{a}$ Faculty of Mathematics and Statistics, Hubei University, Wuhan 430062, China \\ ${ }^{b}$ College of Engineering, Huazhong Agricultural University, Wuhan 430070, China \\ ${ }^{c}$ College of Science, Huazhong Agricultural University, Wuhan 430070, China
}

\begin{abstract}
Learning with Fredholm kernel has attracted increasing attention recently since it can effectively utilize the data information to improve the prediction performance. Despite rapid progress on theoretical and experimental evaluations, its generalization analysis has not been explored in learning theory literature. In this paper, we establish the generalization bound of least square regularized regression with Fredholm kernel, which implies that the fast learning rate $O\left(l^{-1}\right)$ can be reached under mild capacity conditions. Simulated examples show that this Fredholm regression algorithm can achieve the satisfactory prediction performance.
\end{abstract}

Keywords: Fredholm learning, generalization bound, learning rate, data dependent hypothesis spaces

\section{Introduction}

Inspired from Fredholm integral equations, Fredholm learning algorithms are designed recently for density ratio estimation [2] and semi-supervised learning [3]. Fredholm learning can be considered as a kernel method with data-dependent kernel. This kernel usually is called as Fredholm kernel, and can naturally incorporate the data information. Although its empirical performance has been well demonstrated in the previous works, there is no learning theory analysis on generalization bound and learning rate. It is well known that generalization ability and learning rate are important measures to evaluate the learning algorithm [8, 18, 17]. In this paper, we focus on this theoretical theme for regularized least square regression with Fredholm kernel.

In learning theory literature, extensive studies have been established for least square regression with regularized kernel methods, e.g., [12, 13, 16]. Although the Fredholm learning in [3] also can be considered as a regularized kernel method, there are two key features: one is that Fredholm kernel is associated with the "inner" kernel and the "outer" kernel simultaneously, the other is that for the prediction function is double

\footnotetext{
${ }^{*}$ Corresponding author.

Email addresses: tyf3122@163.com(Yanfang Tao), yuanpeipei@webmail.hzau.edu.cn (Peipei Yuan), biqin. song@mail.hzau.edu.cn (Biqin Song)
} 
data-dependent. These characteristics induce the additional difficulty on learning theory analysis. To overcome the difficulty of generalization analysis, we introduce novel stepping-stone functions and establish the decomposition on excess generalization error. The generalization bound is estimated in terms of the capacity conditions on the hypothesis spaces associated with the "inner" kernel and the "outer" kernel, respectively. In particular, the derived result implies that fast learning rate with $O\left(l^{-1}\right)$ can be reached with proper parameter selection, where $l$ is the number of labeled data. To best of our knowledge, this is the first discussion on generalization error analysis for learning with Fredholm kernel.

The rest of this paper is organized as follows. Regression algorithm with Fredholm kernel is introduced in Section 2 and its generalization analysis is presented in Section 3 The proofs of main results are listed in Section 4 Simulated examples are provided in Section 5 and a brief conclusion is summarized in Section 6

\section{Regression with Fredholm kernel}

Let $\mathcal{X} \subset \mathbb{R}^{d}$ be a compact input space and $\mathcal{Y} \subset[-M, M]$ for some constant $M>0$. The labeled data $\mathbf{z}=\left\{z_{i}\right\}_{i=1}^{l}=\left\{\left(x_{i}, y_{i}\right)\right\}_{i=1}^{l}$ are drawn independently from a distribution $\rho$ on $\mathcal{Z}:=\mathcal{X} \times \mathcal{Y}$ and the unlabeled data $\left\{x_{l+j}\right\}_{j=1}^{u}$ are derived random independently according to the marginal distribution $\rho_{\mathcal{X}}$ on $\mathcal{X}$. Given $\mathbf{z}, \mathbf{x}=\left\{x_{i}\right\}_{i=1}^{l+u}$, the main purpose of semi-supervised regression is to find a good approximation of the regression function

$$
f_{\rho}(x)=\int_{y} y d \rho(y \mid \mathcal{X})=\underset{f}{\arg \min } \int_{\mathcal{Z}}(y-f(x))^{2} d \rho(x, y) .
$$

In learning theory,

$$
\mathcal{E}(f):=\int_{\mathcal{Z}}(y-f(x))^{2} d \rho(x, y)
$$

and its discrete version

$$
\mathcal{E}_{\mathbf{z}}(f):=\frac{1}{l} \sum_{i=1}^{l}\left(y-f\left(x_{i}\right)\right)^{2}
$$

are called as the expected risk and the empirical risk of function $f: \mathcal{X} \rightarrow \mathbb{R}$, respectively.

Let $w\left(x, x^{\prime}\right)$ be a continuous bounded function on $X^{2}$ with $\omega:=\sup _{x, x^{\prime}} w\left(x, x^{\prime}\right)<\infty$. Define the integral operator $L_{w}$ as

$$
L_{w} f(x)=\int_{X} w(x, t) f(t) d \rho_{X}(t), \forall f \in L_{\rho_{X}}^{2},
$$

where $L_{\rho_{X}}^{2}$ is the space of square-integrable functions.

Let $\mathcal{H}_{K}$ be a reproducing kernel Hilbert space (RKHS) associated with Mercer kernel $K: X^{2} \rightarrow \mathbb{R}$. Denote $\|\cdot\|_{K}$ as the corresponding norm of $\mathcal{H}_{K}$ and assume the upper bound $\kappa:=\sup _{x, x^{\prime} \in \mathcal{X}} K\left(x, x^{\prime}\right)<\infty$. 
If choose $L_{w} \mathcal{H}=\left\{L_{w} f, f \in \mathcal{H}_{K}\right\}$ as the hypothesis space, the learning problem can be considered as to solve the Fredhom integral equation $L_{w} f(x)=y$. Sine the distribution $\rho$ is unknown, we consider the empirical version of $L_{w} f$ associated with $\mathbf{x}=\left\{x_{i}\right\}_{i=1}^{l+u}$, which is defined as

$$
L_{w, \mathbf{x}} f(x)=\frac{1}{l+u} \sum_{i=1}^{l+u} w\left(x, x_{i}\right) f\left(x_{i}\right) .
$$

In the Fredholm learning framework, the prediction function is constructed from the data dependent hypothesis space

$$
L_{w, \mathbf{x}} \mathcal{H}=\left\{L_{w, \mathbf{x}} f, f \in \mathcal{H}_{K}\right\} .
$$

Given $\mathbf{z}, \mathbf{x}$, least-square regression with Fredholm kernel (LFK) can be formulated as the following optimization

$$
f_{\mathbf{z}}:=f_{\mathbf{z}, \mathbf{x}}=\underset{f \in \mathcal{H}_{K}}{\arg \min }\left\{\mathcal{E}_{\mathbf{z}}\left(L_{w, \mathbf{x}} f\right)+\lambda\|f\|_{K}^{2}\right\},
$$

where $\lambda>0$ is a regularization parameter.

Remark 1. Equation (17) can be considered as a discrete and regularized version of the Fredholm integral equation $L_{w} f=y$. When $w$ is the $\delta$-function, (1) becomes the regularized least square regression in RKHS

$$
\tilde{f}_{\mathbf{z}}=\underset{f \in \mathcal{H}_{K}}{\arg \min }\left\{\mathcal{E}_{\mathbf{z}}(f)+\lambda\|f\|_{K}^{2}\right\} .
$$

When $\mathbf{x}=\left\{x_{i}\right\}_{i=1}^{l}$ and replacing $\|f\|_{K}^{2}$ with $\sum_{i=1}^{l}\left|f\left(x_{i}\right)\right|^{q}, q=1,2$, (1) is equivalent to the data-dependent coefficient regularization

$$
\tilde{f}_{\mathbf{z}}(x)=\sum_{i=1}^{l} \alpha_{\mathbf{z}, i} w\left(x, x_{i}\right),
$$

where

$$
\alpha_{\mathbf{z}}=\underset{\alpha \in \mathbb{R}^{l}}{\arg \min }\left\{\mathcal{E}_{\mathbf{z}}\left(\sum_{i=1}^{l} \alpha_{i} w\left(\cdot, x_{i}\right)\right)+\lambda \sum_{i=1}^{l}\left|\alpha_{i}\right|^{q}\right\} .
$$

It is well known that (2) and (3) have been studied extensively in learning literatures, see, e.g. [10, 12, 13]. These results relied on error analysis techniques for data independent hypothesis space [8, 9, 17] and data dependent hypothesis space [4, 13, 14, 10], respectively. Therefore, the Fredholm learning provides a novel framework for regression related with the data independent space $\mathcal{H}_{K}$ and the data dependent hypothesis space $L_{w, \mathbf{x}} \mathcal{H}$ simultaneously.

Remark 2. Equation (1) involves the "inner" kernel $K$ and the "outer" kernel $w$. Denote

$$
\hat{K}\left(x, x^{\prime}\right)=\frac{1}{(l+u)^{2}} \sum_{i, j=1}^{l+u} w\left(x, x_{i}\right) K\left(x_{i}, x_{j}\right) w\left(x, x_{j}\right),
$$


$\hat{\mathbf{K}}=\left(\hat{K}\left(x_{i}, x_{j}\right)\right)_{i, j=1}^{l}$, and $\mathbf{Y}=\left(y_{1}, \cdots, y_{l}\right)^{T}$. It has been demonstrated in [3] that

$$
L_{w, \mathbf{x}} f_{\mathbf{z}}(x)=\frac{1}{l+u} \sum_{i=1}^{l+u} w\left(x, x_{i}\right) f_{\mathbf{z}}\left(x_{i}\right)=\sum_{s=1}^{l} \hat{K}\left(x, x_{s}\right) \alpha_{s},
$$

where $\alpha=\left(\alpha_{1}, \cdots, \alpha_{l}\right)^{T}=(\hat{\mathbf{K}}+\lambda I)^{-1} \mathbf{Y}$. Therefore, Fredholm regression in (1) can be implemented efficiently and the data-dependent kernel $\hat{K}\left(x, x^{\prime}\right)$ is called Fredholm kernel in [3].

\section{Generalization bound}

To provide the estimation on the excess risk, we introduce some conditions on the hypothesis space capacity and the approximation ability of Fredholm learning framework.

For $R>0$, denote

$$
B_{R}=\left\{f \in \mathcal{H}_{K}:\|f\|_{K} \leq R\right\}
$$

and

$$
\tilde{B}_{R}=\left\{f=\sum_{i=1}^{l+u} \alpha_{i} w\left(\cdot, u_{i}\right): \sum_{i=1}^{l+u}\left|\alpha_{i}\right| \leq R, u_{i} \in \mathcal{X}\right\} .
$$

For any $\varepsilon>0$ and function space $\mathcal{F}$, denote $\mathcal{N}_{\infty}(\mathcal{F}, \varepsilon)$ as the covering number with $\ell_{\infty}$-metric.

Assumption 1. (Capacity condition) For the "inner" kernel K and the "outer" kernel $w$, there exists positive constants $s$ and $p$ such that for any $\varepsilon>0, \log \mathcal{N}_{\infty}\left(B_{1}, \varepsilon\right) \leq$ $c_{s, K} \varepsilon^{-s}$ and $\log \mathcal{N}_{\infty}\left(\tilde{B}_{1}, \varepsilon\right) \leq c_{p, w} \varepsilon^{-p}$, where $c_{s, K}, c_{p, w}>0$ are constants independent of $\varepsilon$.

It is worthy notice that the capacity condition has been well studied in [8, 9, 12]. In particular, this condition holds true when setting the "inner" and "outer" kernels as Gaussian kernel.

For a function $f: \mathcal{X} \rightarrow \mathbb{R}$ and $q \in[1,+\infty)$, denote the $L^{q}$-norm on $\mathcal{X}$ as

$$
\|f\|_{q}:=\|f\|_{L_{\rho_{X}}^{q}}=\left(\int_{\mathcal{X}}|f(x)|^{q} d \rho_{\mathcal{X}}(x)\right)^{\frac{1}{q}} .
$$

Define the data independent regularized function

$$
f_{\lambda}=\underset{f \in \mathcal{H}_{K}}{\arg \min }\left\{\left\|L_{w} f-f_{\rho}\right\|_{2}^{2}+\lambda\|f\|_{K}^{2}\right\} .
$$

The predictor associated with $f_{\lambda}$ is

$$
L_{w} f_{\lambda}=\int_{\mathcal{X}} w(x, t) f_{\lambda}(t) d \rho_{\mathcal{X}}(t)
$$

and the approximation ability of Fredholm scheme in $\mathcal{H}_{K}$ is characterized by

$$
D(\lambda)=\mathcal{E}\left(L_{w} f_{\lambda}\right)-\mathcal{E}\left(f_{\rho}\right)+\lambda\left\|f_{\lambda}\right\|_{K}^{2} .
$$


Assumption 2. (Approximation condition) There exists a constant $\beta \in(0,1]$ such that

$$
D(\lambda) \leq c_{\beta} \lambda^{\beta}, \quad \forall \lambda>0,
$$

where $c_{\beta}$ is a positive constant independent of $\lambda$.

This approximation condition relies on the regularity of $f_{\rho}$, and has been investigated extensively in [9, 13, 7]. To get tight estimation, we introduce the projection operator

$$
\pi(f)(x)= \begin{cases}M, & \text { if } f(x)>M \\ f(x), & \text { if }|f(x)| \leq M \\ -M, & \text { if } f(x)<-M .\end{cases}
$$

It is a position to present the generalization bound.

Theorem 1. Under Assumptions 1 and 2, there exists

$$
\mathcal{E}\left(\pi\left(L_{w, \mathbf{x}} f_{\mathbf{z}}\right)\right)-\mathcal{E}\left(f_{\rho}\right) \leq c \log ^{2}(6 / \delta)\left(\lambda^{-\frac{s}{2+s}} l^{-\frac{s}{2+s}}+\lambda^{\beta}+\lambda^{\beta-1} l^{-\frac{s}{2+p}}\right),
$$

where $c$ is a positive constant independent of $l, \lambda, \delta$

The generalization bound in Theorem 1 depends on the capacity condition, the approximation condition, the regularization parameter $\lambda$, and the number of labeled data. In particular, the labeled data is the key factor on the excess risk without the additional assumption on the marginal distribution. This observation is consistent with the previous analysis for semi-supervised learning [1, 6].

To understand the learning rate of Fredholm regression, we present the following result where $\lambda$ is chosen properly.

Theorem 2. Under Assumptions $\square$ and 2 for any $0<\delta<1$, with confidence $1-\delta$, there exists some positive constant $\tilde{c}$ such that

$$
\mathcal{E}\left(\pi\left(L_{w, \mathbf{x}} f_{\mathbf{z}}\right)\right)-\mathcal{E}\left(f_{\rho}\right) \leq \tilde{c} \log ^{2}(6 / \delta) l^{-\theta},
$$

where

$$
\theta= \begin{cases}\min \left\{\frac{2 \beta}{2+p}, \frac{2}{2+s}-\frac{2 s}{2+s(2+p)}\right\}, & \lambda=l^{-\frac{2}{2+p}} ; \\ \min \left\{\frac{2 \beta}{2 \beta+s \beta+s}, \frac{(2 \beta+s \beta+s)(\beta-1)}{2+s}-\frac{2}{2+p}\right\}, & \lambda=l^{-\frac{2}{2 \beta+s \beta+s}} .\end{cases}
$$

Theorem 2 tells us that Fredholm regression has the learning rate with polynomial decay. When $s=p$, there exists some constant $\bar{c}>0$ such that

$$
\mathcal{E}\left(\pi\left(L_{w, \mathbf{x}} f_{\mathbf{z}}\right)\right)-\mathcal{E}\left(f_{\rho}\right) \leq \bar{c} \log (6 / \delta) l^{-\theta}
$$

with confidence $1-\delta$, where

$$
\theta= \begin{cases}\frac{2 \beta}{2+s}, & \beta \in\left(0, \frac{2}{2+s}\right] \\ \frac{2 \beta}{s+2 \beta+s \beta}, & \beta \in\left(\frac{2}{2+s},+\infty\right] .\end{cases}
$$


and the rate is derived by setting

$$
\lambda= \begin{cases}l^{-\frac{2}{2+s}}, & \beta \in\left(0, \frac{2}{2+s}\right] ; \\ l^{-\frac{2}{s+2 \beta+s \beta}}, & \beta \in\left(\frac{2}{2+s},+\infty\right] .\end{cases}
$$

This learning rate can be arbitrarily close to $O\left(l^{-1}\right)$ as $s$ tends to zero, which is regarded as the fastest learning rate for regularized regression in the learning theory literature. This result verifies the LFK in (1) inherits the theoretical characteristics of least square regularized regression in RKHS [9, 16] and in data dependent hypothesis spaces [12, 14].

\section{Error analysis}

We first present the decomposition on the excess risk $\mathcal{E}\left(\pi\left(L_{w, \mathbf{x}} f_{\mathbf{z}}\right)\right)-\mathcal{E}\left(f_{\rho}\right)$, and then establish the upper bounds of different error terms.

\subsection{Error decomposition}

According to the definitions of $f_{\mathbf{z}}, f_{\lambda}$, we can get the following error decomposition.

Proposition 1. For $f_{\mathbf{z}}$ defined in (1), there holds

$$
\mathcal{E}\left(\pi\left(L_{w, \mathbf{x}} f_{\mathbf{z}}\right)\right)-\mathcal{E}\left(f_{\rho}\right) \leq E_{1}+E_{2}+E_{3}+D(\lambda)
$$

where

$$
\begin{aligned}
& E_{1}=\mathcal{E}\left(\pi\left(L_{w, \mathbf{x}} f_{\mathbf{z}}\right)\right)-\mathcal{E}\left(f_{\rho}\right)-\left(\mathcal{E}_{\mathbf{z}}\left(\pi\left(L_{w, \mathbf{x}} f_{\mathbf{z}}\right)\right)-\mathcal{E}_{\mathbf{z}}\left(f_{\rho}\right)\right), \\
& E_{2}=\mathcal{E}_{\mathbf{z}}\left(L_{w, \mathbf{x}} f_{\lambda}\right)-\mathcal{E}_{\mathbf{z}}\left(f_{\rho}\right)-\left(\mathcal{E}\left(L_{w, \mathbf{x}} f_{\lambda}\right)-\mathcal{E}\left(f_{\rho}\right)\right),
\end{aligned}
$$

and

$$
E_{3}=\mathcal{E}\left(L_{w, \mathbf{x}} f_{\lambda}\right)-\mathcal{E}\left(L_{w} f_{\lambda}\right)
$$

Proof: By introducing the middle function $L_{w, \mathbf{x}} f_{\lambda}$, we get

$$
\begin{aligned}
& \mathcal{E}\left(\pi\left(L_{w, \mathbf{x}} f_{\mathbf{z}}\right)\right)-\mathcal{E}\left(f_{\rho}\right) \\
\leq \quad & \mathcal{E}\left(\pi\left(L_{w, \mathbf{x}} f_{\mathbf{z}}\right)\right)-\mathcal{E}_{\mathbf{z}}\left(\pi\left(L_{w, \mathbf{x}} f_{\mathbf{z}}\right)\right)+\left[\mathcal{E}_{\mathbf{z}}\left(L_{w, \mathbf{x}} f_{\mathbf{z}}\right)+\lambda\left\|f_{\mathbf{z}}\right\|_{K}^{2}-\left(\mathcal{E}_{\mathbf{z}}\left(L_{w, \mathbf{x}} f_{\lambda}\right)+\lambda\left\|f_{\lambda}\right\|_{K}^{2}\right)\right] \\
& +\mathcal{E}_{\mathbf{z}}\left(L_{w, \mathbf{x}} f_{\lambda}\right)-\mathcal{E}\left(L_{w, \mathbf{x}} f_{\lambda}\right)+\mathcal{E}\left(L_{w, \mathbf{x}} f_{\lambda}\right)-\mathcal{E}\left(L_{w} f_{\lambda}\right)+\mathcal{E}\left(L_{w} f_{\lambda}\right)-\mathcal{E}\left(f_{\rho}\right)+\lambda\left\|f_{\lambda}\right\|_{K}^{2} \\
\leq & E_{1}+E_{2}+E_{3}+D(\lambda)
\end{aligned}
$$

where the last inequality follows from the definition $f_{\mathbf{z}}$. This completes the proof.

In learning theory, $E_{1}, E_{2}$ are called the sample error, which describe the difference between the empirical risk and the expected risk. $E_{3}$ is called the hypothesis error which reflects the divergence of expected risks between the data independent function $L_{w} f_{\lambda}$ and data dependent function $L_{w, \mathbf{x}} f_{\lambda}$. 


\subsection{Estimates of sample error}

We introduce the concentration inequality in [15] to measure the divergence between the empirical risk and the expected risk.

Lemma 1. Let $\mathcal{F}$ be a measurable function set on $\mathcal{Z}$. Assume that, for any $f \in \mathcal{F}$, $\|f\|_{\infty} \leq B$ and $E\left(f^{2}\right) \leq c E f$ for some positive constants $B$, c. If for some $a>0$ and $s \in(0,2), \log \mathcal{N}_{2}(\mathcal{F}, \varepsilon) \leq a \varepsilon^{-s}$ for any $\varepsilon>0$, then there exists a constant $c_{s}$ such that for any $\delta \in(0,1)$,

$$
\left|E f-\frac{1}{m} \sum_{i=1}^{m} f\left(z_{i}\right)\right| \leq c_{s} \max \left\{c^{\frac{2-s}{2+s}}, B^{\frac{2-s}{2+s}}\right\}\left(\frac{a}{m}\right)^{\frac{2}{2+s}}+\frac{1}{2} E f+\frac{(2 c+18 B) \log (1 / \delta)}{m}
$$

with confidence at least $1-2 \delta$.

To estimate $E_{1}$, we consider the function set containing $f_{\mathbf{z}}$ for any $\mathbf{z} \in \mathcal{Z}^{l}, \mathbf{u} \in \mathcal{X}^{u}$. The definition $f_{\mathbf{z}}$ in (1) tells us that $\left\|f_{\mathbf{z}}\right\|_{K} \leq \frac{M}{\sqrt{\lambda}}$. Hence, $\forall \mathbf{z} \in \mathcal{Z}^{l}, f_{\mathbf{z}} \in B_{R}$ with $R=\frac{M}{\sqrt{\lambda}}$ and $\left\|f_{\mathbf{z}}\right\|_{\infty} \leq \frac{\kappa M}{\sqrt{\lambda}}$.

Proposition 2. Under Assumption 1 for any $0<\delta<1$,

$$
E_{1} \leq \frac{1}{2}\left(\mathcal{E}\left(\pi\left(L_{w, \mathbf{x}} f_{\mathbf{z}}\right)\right)-\mathcal{E}\left(f_{\rho}\right)\right)+c_{1} \lambda^{-\frac{s}{2+s}} m^{-\frac{s}{2+s}}+176 M^{2} l^{-1} \log (1 / \delta)
$$

with confidence $1-\delta$.

Proof: For $f \in B_{R}, \mathbf{z} \in \mathcal{Z}^{l}, \mathbf{x} \in X^{l+u}$, denote

$$
G_{R}=\left\{g(z)=\left(y-\pi\left(L_{w, \mathbf{x}} f\right)\right)^{2}-\left(y-f_{\rho}(x)\right)^{2}\right\} .
$$

For any $z \in \mathcal{Z}$,

$$
|g(z)| \leq\left|2 y-\pi\left(L_{w, \mathbf{x}} f\right)(x)-f_{\rho}(x) \| \pi\left(L_{w, \mathbf{x}} f\right)-f_{\rho}(x)\right| \leq 8 M^{2} .
$$

Moreover,

$$
E g^{2} \leq 16 M^{2} E\left(\pi\left(L_{w, \mathbf{x}} f\right)(x)-f_{\rho}(x)\right)^{2}=16 M^{2} E g .
$$

For any $f_{1}, f_{2} \in B_{R}$, there exists

$$
\left|g_{1}(z)-g_{2}(z)\right| \leq \frac{4 M}{l+u}\left|\sum_{i=1}^{l+u}\left(f_{1}\left(x_{i}\right)-f_{2}\left(x_{i}\right)\right) w\left(x, x_{i}\right)\right| \leq 4 M \omega\left\|f_{1}-f_{2}\right\|_{\infty} .
$$

This relation implies that

$$
\log \mathcal{N}_{\infty}\left(G_{R}, \varepsilon\right) \leq \log \mathcal{N}_{\infty}\left(B_{1}, \frac{\varepsilon}{4 M \omega R}\right) \leq c_{s, K}(4 M \omega R)^{s} \varepsilon^{-s},
$$

where the last inequality from Assumption 1 
Applying the above estimates to Lemma1, we derive that

$$
\begin{aligned}
& E g-\frac{1}{l} \sum_{i=1}^{l} g\left(z_{i}\right) \\
\leq & \frac{1}{2} E g+\max \left\{16 M^{2} \omega, 8 M^{2}\right\}^{\frac{2-s}{2+s}} c_{s, K}^{\frac{2}{2+s}}(4 M \omega R)^{\frac{2 s}{2+s}} l^{-\frac{2}{2+s}}+176 M^{2} l^{-1} \log (1 / \delta)
\end{aligned}
$$

with confidence $1-\delta$.

Considering $f_{\mathbf{z}} \in B_{R}$ with $R=\frac{M}{\sqrt{\lambda}}$, we obtain the desired result.

Proposition 3. Under Assumption 1, with confidence $1-4 \delta$, there holds

$$
E_{2} \leq \frac{1}{2} E_{3}+\frac{1}{2} D(\lambda)+c_{2} D(\lambda) \lambda^{-1} l^{-\frac{2}{2+p}} \log (1 / \delta),
$$

where $c_{2}$ is a positive constant independent of $\lambda, m, \delta$.

Proof: Denote

$$
\mathcal{G}=\left\{g_{\mathbf{v}, \lambda}: g_{\mathbf{v}, \lambda}(x)=L_{w, \mathbf{v}} f_{\lambda}(x), x, v_{i} \in \mathcal{X}\right\}
$$

From the definition $f_{\lambda}$, we can deduce that $\forall g \in \mathcal{G}, g \in \tilde{B}_{R}$ with $R=\omega \kappa \sqrt{\frac{D(\lambda)}{\lambda}}$. For $z \in \mathcal{Z}, \mathbf{v} \in \mathcal{X}^{l+u}$, define

$$
\mathcal{H}=\left\{h(z)=\left(y-L_{w, \mathbf{v}} f_{\lambda}(x)\right)^{2}-\left(y-f_{\rho}(x)\right)^{2}\right\} .
$$

It is easy to check that for any $z \in \mathcal{Z}$

$$
\begin{aligned}
|h(z)| & =\left|2 y-L_{w, \mathbf{v}} f_{\lambda}(x)-f_{\rho}(x)\right| \cdot\left|L_{w, \mathbf{v}} f_{\lambda}(x)-f_{\rho}(x)\right| \\
& \leq\left(3 M+\omega\left\|f_{\lambda}\right\|_{\infty}\right)^{2} \leq\left(3 M+\omega \kappa \sqrt{\frac{D(\lambda)}{\lambda}}\right)^{2} .
\end{aligned}
$$

Then,

$$
\begin{aligned}
E h^{2} & =E\left(2 y-L_{w, \mathbf{v}} f_{\lambda}(x)-f_{\rho}(x)\right)^{2}\left(L_{w, \mathbf{v}} f_{\lambda}(x)-f_{\rho}(x)\right)^{2} \\
& \leq\left(3 M+w k \sqrt{\frac{D(\lambda)}{\lambda}}\right)^{2} E h .
\end{aligned}
$$

For any $\mathbf{u}, \mathbf{v} \in \mathcal{X}^{l+u}$, there exists

$$
\begin{aligned}
\left\|h_{1}-h_{2}\right\|_{\infty} & =\sup _{z}\left|\left(y-L_{w, \mathbf{u}} f_{\lambda}(x)\right)^{2}-\left(y-L_{w, \mathbf{v}} f_{\lambda}(x)\right)^{2}\right| \\
& \leq 2\left(M+\omega \kappa \sqrt{\frac{D(\lambda)}{\lambda}}\right)\left\|L_{w, \mathbf{u}} f_{\lambda}-L_{w, \mathbf{v}} f_{\lambda}\right\|_{\infty} \\
& =2\left(M+\omega \kappa \sqrt{\frac{D(\lambda)}{\lambda}}\right)\left\|g_{\mathbf{u}, \lambda}-g_{\mathbf{v}, \lambda}\right\|_{\infty} .
\end{aligned}
$$


Then from Assumption1

$$
\log \mathcal{N}_{\infty}(\mathcal{H}, \varepsilon) \leq \log \mathcal{N}_{\infty}\left(\tilde{B}_{R}, \frac{\varepsilon}{2\left(M+\omega \kappa \sqrt{\frac{D(\lambda)}{\lambda}}\right)}\right) \leq 4 c_{p, w}\left(M+\omega \kappa \sqrt{\frac{D(\lambda)}{\lambda}}\right)^{2 p} \varepsilon^{-p} .
$$

Combining (5)-(7) with Lemma1, we get with confidence $1-\delta$

$$
\begin{aligned}
E_{2} \leq & \frac{1}{2}\left(\mathcal{E}\left(L_{w, \mathbf{x}} f_{\lambda}\right)-f_{\rho}\right)+\left(M+\omega \kappa \sqrt{\frac{D(\lambda)}{\lambda}}\right)^{2} l^{-\frac{2}{2+p}} \\
& \cdot c_{p}\left(4 c_{p, w}\right)^{\frac{2}{2+p}}+\frac{20\left(3 M+\omega \kappa \sqrt{\frac{D(\lambda)}{\lambda}}\right) \log (1 / \delta)}{l} .
\end{aligned}
$$

Considering $\mathcal{E}\left(L_{w, \mathbf{x}} f_{\lambda}\right)-\mathcal{E}\left(f_{\rho}\right) \leq E_{3}+D(\lambda)$, we get the desired result.

\subsection{Estimate of hypothesis error}

The following concentration inequality with values in Hilbert space can be found in [11], which is used in our analysis.

Lemma 2. Let $\mathcal{H}$ be a Hilbert space and $\xi$ be independent random variable on $Z$ with values in $\mathcal{H}$. Assume that $\|\xi\|_{\mathcal{H}} \leq \tilde{M}<\infty$ almost surely. Let $\left\{z_{i}\right\}_{i=1}^{m}$ be independent random samples from $\rho$. Then, for any $\delta \in(0,1)$,

$$
\left\|\frac{1}{m} \sum_{i=1}^{m} \xi\left(z_{i}\right)-E \xi\right\|_{\mathcal{H}} \leq \frac{2 \tilde{M} \log \left(\frac{1}{\delta}\right)}{M}+\sqrt{\frac{2 E\|\xi\|_{\mathcal{H}}^{2} \log \left(\frac{1}{\delta}\right)}{m}}
$$

holds true with confidence $1-\delta$.

Now we turn to estimate $E_{3}$, which reflects the affect of inputs $\mathbf{x}=\left\{x_{i}\right\}_{i=1}^{l+u}$ to the regularization function $f_{\lambda}$.

Proposition 4. For any $0<\delta<1$, with confidence $1-\delta$, there holds

$$
E_{3} \leq 24 \omega^{2} \kappa^{2} \log ^{2}\left(\frac{1}{\delta}\right) D(\lambda) \lambda^{-1}(l+u)^{-1}+D(\lambda) .
$$

Proof: Note that

$$
\begin{aligned}
& \mathcal{E}\left(L_{w, \mathbf{x}} f_{\lambda}\right)-\mathcal{E}\left(L_{w} f_{\lambda}\right) \\
\leq & \left\|L_{w, \mathbf{x}} f_{\lambda}-L_{w} f_{\lambda}\right\|_{2} \cdot\left(\left\|L_{w, \mathbf{x}} f_{\lambda}-f_{\rho}\right\|_{2}+\left\|L_{w} f_{\lambda}-f_{\rho}\right\|_{2}\right) \\
\leq & \left\|L_{w, \mathbf{x}} f_{\lambda}-L_{w} f_{\lambda}\right\|_{2}\left(\left\|L_{w, \mathbf{x}} f_{\lambda}-L_{w} f_{\lambda}\right\|_{2}+2\left\|L_{w} f_{\lambda}-f_{\rho}\right\|_{2}\right) \\
\leq & 2\left\|L_{w, \mathbf{x}} f_{\lambda}-L_{w} f_{\lambda}\right\|_{2}^{2}+\left\|L_{w} f_{\lambda}-f_{\rho}\right\|_{2}^{2} \\
\leq & 2\left\|L_{w, \mathbf{x}} f_{\lambda}-L_{w} f_{\lambda}\right\|_{2}^{2}+D(\lambda) .
\end{aligned}
$$

Denote $\xi\left(x_{i}\right)=f_{\lambda}\left(x_{i}\right) w\left(\cdot, x_{i}\right)$, which is continuous and bounded function on $\mathcal{X}$. Then

$$
L_{w, \mathbf{x}} f_{\lambda}=\frac{1}{l+u} \sum_{i=1}^{l+u} \xi\left(x_{i}\right)
$$


and

$$
L_{w} f_{\lambda}=\int w(\cdot, t) f_{\lambda}(t) d \rho_{\mathcal{X}}(t)=E \xi .
$$

We can deduce that $\|\xi\|_{2} \leq \omega\left\|f_{\lambda}\right\|_{\infty} \leq \omega \kappa\left\|f_{\lambda}\right\|_{K}$ and $E\|\xi\|_{2}^{2} \leq \omega^{2} \kappa^{2}\left\|f_{\lambda}\right\|_{K}^{2}$. From Lemma2, for any $0<\delta<1$, there holds with confidence $1-\delta$

$$
\left\|L_{w, \mathbf{x}} f_{\lambda}-L_{w} f_{\lambda}\right\|_{2} \leq \frac{2 \omega \kappa\left\|f_{\lambda}\right\|_{K} \log \left(\frac{1}{\delta}\right)}{l+u}+\sqrt{\frac{2 \log \left(\frac{1}{\delta}\right)}{l+u}} \omega \kappa\left\|f_{\lambda}\right\|_{K} .
$$

Combining (8) and (9), we get with confidence $1-\delta$,

$$
\begin{aligned}
E_{3} & \leq 2\left(\frac{2 \omega \kappa\left\|f_{\lambda}\right\|_{K} \log \left(\frac{1}{\delta}\right)}{l+u}+\omega \kappa\left\|f_{\lambda}\right\|_{K} \sqrt{\frac{2 \log \left(\frac{1}{\delta}\right)}{l+u}}\right)^{2}+D(\lambda) \\
& \leq \frac{16 \omega^{2} \kappa^{2}\left\|f_{\lambda}\right\|_{K}^{2} \log ^{2}\left(\frac{1}{\delta}\right)}{(l+u)^{2}}+\frac{8 \omega^{2} \kappa^{2}\left\|f_{\lambda}\right\|_{K}^{2} \log \left(\frac{1}{\delta}\right)}{l+u}+D(\lambda) .
\end{aligned}
$$

Then, the desired result follows from $\left\|f_{\lambda}\right\|_{K}^{2} \leq \frac{D(\lambda)}{\lambda}$.

\subsection{Proofs of Theorem 1 and 2}

Proof of Theorem 1: Combining the estimations in Propositions 1-4, we get with confidence $1-6 \delta$,

$$
\begin{aligned}
& \mathcal{E}\left(\pi\left(L_{w, \mathbf{x}} f_{\mathbf{z}}\right)\right)-\mathcal{E}\left(f_{\rho}\right) \\
\leq & \frac{1}{2}\left(\mathcal{E}\left(\pi\left(L_{w, \mathbf{x}} f_{\mathbf{z}}\right)\right)-\mathcal{E}\left(f_{\rho}\right)\right)+c_{1} \lambda^{-\frac{s}{2+s}} l^{-\frac{2}{2+s}}+176 M^{2} l^{-1} \log \left(\frac{1}{\delta}\right) \\
& +3 D(\lambda)+c_{2} D(\lambda) \lambda^{-1} l^{-\frac{2}{2+p}} \log \left(\frac{1}{\delta}\right)+\frac{36 w^{2} k^{2} \log ^{2}\left(\frac{1}{\delta}\right)}{l+u} \frac{D(\lambda)}{\lambda} .
\end{aligned}
$$

Considering $u>0$, for $0<\delta<1$, we have with confidence $1-6 \delta$

$$
\mathcal{E}\left(\pi\left(L_{w, \mathbf{x}} f_{\mathbf{z}}\right)\right)-\mathcal{E}\left(f_{\rho}\right) \leq c \log ^{2}\left(\frac{1}{\delta}\right)\left[\lambda^{-\frac{s}{2+s}} l^{-\frac{s}{2+s}}+\lambda^{\beta}+\lambda^{\beta-1} l^{-\frac{2}{2+p}}\right],
$$

where $c$ is a constant independent of $l, \lambda, \delta$.

Proof of Theorem 2: When setting $\lambda^{\beta}=\lambda^{\beta-1} l^{-\frac{2}{2+p}}$, we obtain $\lambda=l^{-\frac{2}{2+p}}$. Then, Theorem11implies that

$$
\mathcal{E}\left(\pi\left(L_{w, \mathbf{x}} f_{\mathbf{z}}\right)\right)-\mathcal{E}\left(f_{\rho}\right) \leq 3 c \log ^{2}\left(\frac{1}{\delta}\right) l^{-\min \left\{\frac{2 \beta}{2+p}, \frac{2}{2+s}-\frac{2 s}{(2+s)(2+p)}\right\}} .
$$

When setting $\lambda^{\beta}=\lambda^{-\frac{s}{2+s}} l^{-\frac{2}{2+s}}$, we get $\lambda=l^{-\frac{2}{2 \beta+s \beta+s}}$. Then, with confidence $1-6 \delta$

$$
\mathcal{E}\left(\pi\left(L_{w, \mathbf{x}} f_{\mathbf{z}}\right)\right)-\mathcal{E}\left(f_{\rho}\right) \leq 3 c \log ^{2}\left(\frac{1}{\delta}\right) l^{-\min \left\{\frac{2 \beta}{2 \beta+s \beta+s} \frac{(2 \beta+s \beta+s)(\beta-1)}{2+s}+\frac{2}{2+p}\right\}} .
$$

This complete the proof of Theorem 2 . 
Table 1: MSE \pm STD for LFK and SVM with 50 and 100 training samples

\begin{tabular}{c|ccccc}
\hline Function & Number & SVM & LFK 1 & LFK 2 & LFK3 \\
\hline$f_{1}$ & 50 & $0.041 \pm 0.033$ & $0.434 \pm 0.032$ & $0.423 \pm 0.059$ & $\mathbf{0 . 0 3 6} \pm \mathbf{0 . 0 5 3}$ \\
& 300 & $0.044 \pm 0.006$ & $0.419 \pm 0.023$ & $0.404 \pm 0.021$ & $\mathbf{0 . 0 4 2} \pm \mathbf{0 . 0 0 6}$ \\
\hline$f_{2}$ & 50 & $0.075 \pm 0.046$ & $18.52 \pm 1.30$ & $18.7 \pm 1.30$ & $\mathbf{0 . 0 6 0} \pm \mathbf{0 . 0 2 8}$ \\
& 300 & $\mathbf{0 . 0 1 1} \pm 0.006$ & $17.10 \pm 0.941$ & $17.00 \pm 1.35$ & $0.012 \pm \mathbf{0 . 0 0 4}$ \\
\hline$f_{3}$ & 50 & $0.013 \pm 0.012$ & $0.670 \pm 0.034$ & $0.458 \pm 0.082$ & $\mathbf{0 . 0 1 0} \pm \mathbf{0 . 0 0 5}$ \\
& 300 & $0.004 \pm 0.001$ & $0.667 \pm 0.013$ & $0.427 \pm 0.020$ & $\mathbf{0 . 0 0 3} \pm \mathbf{0 . 0 0 1}$ \\
\hline$f_{4}$ & 50 & $0.076 \pm 0.027$ & $0.260 \pm \mathbf{0 . 0 1 2}$ & $0.194 \pm 0.040$ & $\mathbf{0 . 0 7 3} \pm 0.021$ \\
& 300 & $0.039 \pm 0.018$ & $0.251 \pm 0.017$ & $0.158 \pm 0.026$ & $\mathbf{0 . 0 3 2} \pm \mathbf{0 . 0 0 9}$ \\
\hline
\end{tabular}

\section{Empirical studies}

To verify the effectiveness of LFK in (1), we present some simulated examples for the regression problem. The competing method is support vector machine regression (SVM), which has been used extensively used in machine learning community (https://www.csie.ntu.edu.tw/ cjlin/libsvm/). The Gaussian kernel $K(x, t)=$ $\exp \left\{-\frac{\|x-t\|_{2}^{2}}{2 \sigma^{2}}\right\}$ is used for SVM. For LFK in (1), we consider the following "inner" and "outer" kernels:

- LFK1: $w(x, z)=x^{T} z$ and $K(x, z)=\exp \left\{-\frac{\|x-t\|_{2}^{2}}{\sigma^{2}}\right\}$.

- LFK2: $w(x, z)=\exp \left\{-\frac{\|x-t\|_{2}^{2}}{\sigma^{2}}\right\}$ and $K(x, z)=x^{T} z$.

- LFK3: $w(x, z)=\exp \left\{-\frac{\|x-t\|_{2}^{2}}{\sigma^{2}}\right\}$ and $K(x, z)=\exp \left\{-\frac{\|x-t\|_{2}^{2}}{\sigma^{2}}\right\}$.

Here the scale parameter $\sigma$ belongs to $\left[2^{-5}: 2: 2^{5}\right]$ and the regularization parameter belongs to $\left[10^{-5}: 10: 10^{5}\right]$ for LFK and SVM. These parameters are selected by 4 -fold cross validation in this section.

The following functions are used to generate the simulated data:

$$
\begin{aligned}
& f_{1}(x)=\sin \left(\frac{9 \pi}{0.35 x+1}\right), \quad x \in[0,10] \\
& f_{2}(x)=x \cos (x), \quad x \in[0,10] \\
& f_{3}(x)=\min (2|x|-1,1), \quad x \in[-2,2] \\
& f_{4}(x)=\operatorname{sign}(x), \quad x \in[-3,3] .
\end{aligned}
$$

Note that $f_{1}$ is highly oscillatory, $f_{2}$ is smooth, $f_{3}$ is continuous not smooth, and $f_{4}$ is not even continuous. These functions have been used to evaluate regression algorithms in [14].

In our experiment, Gaussian noise $N(0,0.01)$ is added to the data respectively. In each test, we first draw randomly 1000 samples according to the function and noise 


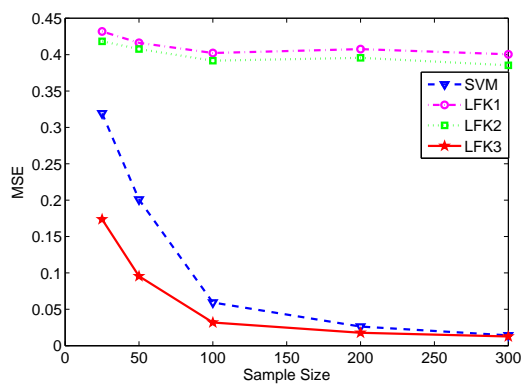

(a)

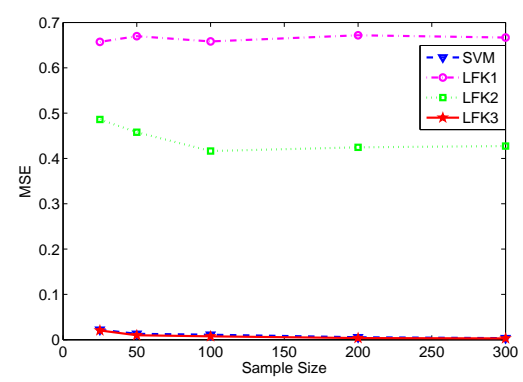

(c)

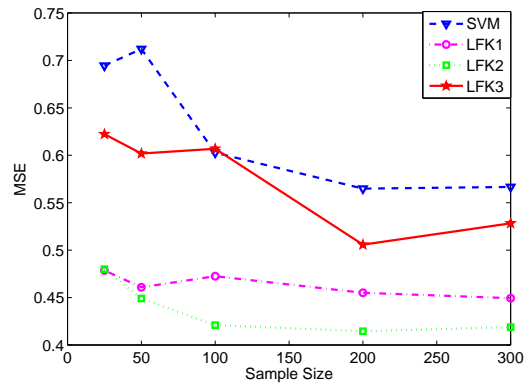

(b)

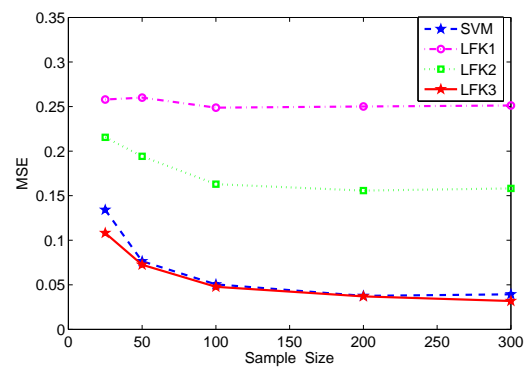

(d)

Figure 1: MSE for Gaussian noise and varying training sample size. (a) $f_{1}$; (b) $f_{2}$; (c) $f_{3}$; (d) $f_{4}$.

distribution, and then obtain a training set randomly with sizes 25, 50, 100, 200, 300 respectively. Three hundred samples are selected randomly as the test set. The Mean Squared Error (MSE) is used to evaluate the regression results on synthetic data. To make the results more convincing, each test is repeated 10 times. Table 1 reports the average MSE and Standard Deviation (STD) with 50 training samples and 300 training samples respectively. Furthermore, we study the impact of the number of training samples on the final regression performance. Figure 1 shows the MSE for learning $f_{1}-$ $f_{4}$ with numbers of training samples. These results illustrate that LFK has competitive performance compared with SVM.

\section{Conclusion}

This paper investigated the generalization performance of regularized least square regression with Fredholm kernel. Generalization bound is presented for the Fredholm learning model, which shows that the fast learning rate with $O\left(l^{-1}\right)$ can be reached. In the future, it is interesting to investigate the leaning performance of ranking [5] with Fredholm kernel. 


\section{Acknowledgments}

The authors would like to thank Prof.Dr.L.Q. Li for his valuable suggestions. This work was supported by the National Natural Science Foundation of China(Grant Nos. 11671161) and the Fundamental Research Funds for the Central Universities (Program Nos. 2662015PY046, 2014PY025).

\section{References}

\section{References}

[1] M. Belkin, P. Niyogi, and V. Sindhwani, "Manifold regularization: A geometric framework for learning from labeled and unlabeled examples," J. Mach. Learn. Res., vol. 7, pp. 2399-2434, 2006.

[2] Q. Que and M. Belkin, "Inverse density as an inverse problem: the fredholm equation approach,” In NIPS, pp. 1484-1492, 2013.

[3] Q. Que, M. Belkin, and Y. Wang, "Learning with Fredholm kernels," In NIPS, pp. 2951-2959, 2014.

[4] H. Chen, Z. Pan, L.Q. Li, Y.Y. Tang, "Learning rates of coefficient-based regularized classifier for density level detection," Neural Computation, vol. 25, no. 4, pp. 1107-1121, 2013.

[5] H. Chen, Y. Tang, L.Q. Li, Y. Yuan, X. Li, and Y.Y. Tang, "Error analysis of stochastic gradient descent ranking," IEEE Trans. Cybern., vol. 43, pp. 898-909, 2013.

[6] H. Chen, Y. Zhou, Y.Y. Tang, L.Q. Li, and Z. Pan, "Convergence rate of semisupervised greedy algorithm," Neural Networks, vol. 44, pp. 44-50, 2013.

[7] H. Chen and L.Q. Li, "Learning rates of multi-kernel regularized regression," Journal of Statistical Planning and Inference, vol. 140, pp. 2562-2568, 2010.

[8] F. Cucker and S. Smale, "On the mathematical foundations of learning, " Bull. Amer. Math. Soc. , vol. 39, no. 39, pp. 1-49, 2002.

[9] F. Cucker and D. X. Zhou, Learning Theory: An Approximation Theory Viewpoint. Cambridge, U. K. : Cambridge Univ. Press, 2007.

[10] Y. Feng, S. Lv, H. Huang, and J. Suykens, "Kernelized elastic net reguularization: generalization bouunds and sparse recovery," Neural Comput., vol. 28, pp. 1-38, 2016.

[11] I. Pinelis, "Optimum bounds for the distribution of martingales in Banach spaces," Ann. Probab., vol. 22, pp. 1679-1706, 1994.

[12] L. Shi, Y. Feng, and D.X. Zhou, "Concentration estimates for learning with $\ell_{1}$ regularizer and data dependent hypothesis spaces," Appl. Comput. Harmon. Anal., vol. 31, no. 2, pp. 286-302, 2011. 
[13] H. Sun and Q. Wu, "Least square regression with indefinite kernels and coefficient regularization," Appl. Comput. Harmon. Anal., vol. 30, no. 1, pp. 96-109, 2011.

[14] H. Sun and Q. Wu, "Sparse representation in kernel machines," IEEE Trans. Neural Netw. Learning Syst., vol. 26, no. 10, 2576-2582, 2015.

[15] Q. Wu, Y. Ying, and D.X. Zhou, "Multi-kernel regularized classfiers," J. Complexity, vol. 23, pp. 108-134, 2007.

[16] Q. Wu, Y.M. Ying, and D.X. Zhou, "Learning rates of least-square regularized regression," Found. Comput. Math., vol. 6, pp. 171-192, 2006.

[17] B. Zou, L.Q. Li, and Z.B. Xu, "The generalization performance of ERM algorithm with strongly mixing observations," Machine Learning, vol. 75, no. 3, pp. 275-295, 2009.

[18] B. Zou, R. Chen, and Z.B. Xu, "Learning performance of Tikhonov regularization algorithm with geometrically beta-mixing observations," Journal of Statistical Planning and Inference, vol. 141, pp. 1077-1087, 2011. 